\title{
Biomass Production and Nutritional Composition of Moringa oleifera under Different Cutting Frequencies and Planting Spacings
}

\author{
Shahzad Maqsood Ahmed Basra ${ }^{{ }^{*}}$, Wasif Nouman ${ }^{*}$, Hafeez-ur-Rehman ${ }^{1}$, Muhammad Usman ${ }^{1}$ and Zill-e-Huma \\ Nazli $^{3}$ \\ ${ }^{1}$ Department of Agronomy, University of Agriculture Faisalabad, Pakistan \\ ${ }^{2}$ Department of Forestry \& Range Management, Bahauddin Zakariya University Multan, Pakistan \\ ${ }^{3}$ Department of Chemistry, GC University for Women, Madina Town, Pakistan \\ *For correspondence: shehzadbasra@gmail.com
}

\begin{abstract}
Moringa (Moringa oleifera L.) is a multipurpose plant with high nutritional composition and can be re-shaped in tree, hedge, fence etc. due to its high re-growth capability and being grown as multi-cut forage. This study investigated the effect of different planting spacing and cutting frequencies on biomass production and nutritional quality of moringa. Seeds of moringa were sown on beds at plant spacing of $15 \mathrm{~cm} \times 30 \mathrm{~cm}$ (narrow) and $15 \times 60 \mathrm{~cm}$ (broad). The cutting frequencies for fresh biomass were (i) $15 \mathrm{~d}$ (ii) $20 \mathrm{~d}$ and (iii) $30 \mathrm{~d}$. Fresh matter yield in $1^{\text {st }}$ and $2^{\text {nd }}$ year was recorded as 6.40 and $7.57 \mathrm{t} \mathrm{ha}{ }^{-1}$, respectively when moringa crop was planted at narrow plant spacing with $30 \mathrm{~d}$ cutting interval followed by cutting interval of 15 and $20 \mathrm{~d}$ at the same planting spacing in year 1 and 2, respectively. However, the growth rate was highest when moringa plants were harvested at $15 \mathrm{~d}$ cutting interval. A significant variation in mineral composition of moringa leaves was also observed during this research. Nitrogen $(6.11 \%)$, potassium $(9.14 \%)$ and ascorbate $\left(89.73 \mu \mathrm{g} \mathrm{g}^{-1}\right)$ were recorded when moringa crop was harvested at $30 \mathrm{~d}$ cutting interval at broad spacing, while phosphorous (3.40\%) was recorded at $20 \mathrm{~d}$ cutting interval, whilst maximum calcium content $(2.53 \%)$ were recorded when the crop was harvested at $30 \mathrm{~d}$ interval at narrow planting spacing. In conclusion, for maximum biomass production with better nutritional composition, moringa should be established as fodder purpose at narrow spacing $(15 \mathrm{~cm} \times 30 \mathrm{~cm})$ with optimum cutting interval time of 30 days. C) 2016 Friends Science Publishers
\end{abstract}

Keywords: Fodder Shortage; Biomass; Cutting intervals; Minerals; Moringa; Planting geometry

\section{Introduction}

Adequate and regular supply of quality fodder is essential for the development of livestock to meet the increasing demands of burgeoning population. Livestock sector is considered as the mainstay of Pakistan's economy and $25-30 \%$ of the population subsistence depends on it (Government of Pakistan, 2014). The prevalence of prolonged dry period directly affects the grasslands and farming areas, which create the scarcity of green fodder for livestock. To maintain reproductive performance and avoid nutritional stress, adequate supply and availability of nutritious fodders is essential during this period (Benavides, 1994; Raziq et al., 2010).

According to national fodder program, cultivated area under fodder has decreased up to $11.6 \%$ during the last two years with shortage of about 28.62 million tons of total digestible nutrients (TDN) and about 1.76 million tons of digestible protein (DP). More than half of animal requirements are met through fodders and crop residues, one third from grazing of rangelands, wastelands, canal banks, road sides and the rest is from crops and their by-products (NARC, 2007). One of the potential strategies to implement proper land usage or increasing the quality and availability of feed during dry season is to grow fodder trees and shrub forages (Pezo, 1991). The fodder trees are gaining more attention due to low maintenance and less input requirements and their capability to provide good quality forage during the periods of food scarcity (Palada, 1996).

Moringa (Moringa oleifera L.) is one of the best nutritious trees, which can provide sufficient fodder for livestock during dry season. It is a fast growing tree with efficient capability of re-growth after pruning and capacity to produce good quality higher leaf biomass per unit area (Foidl et al., 2001; Nouman et al. 2014). Unlike other fodders plants, moringa can grow in all types of soils preferable sandy loam except waterlogged condition, and can tolerate long dry spells up to 6 months during dry season and grow well with annual rainfall between 250-1500 mm per year (HDRA, 2002; Abdulkarim et al.,

To cite this paper: S.M.A. Basra, W. Nouman, H.U. Rehman, M. Usman and Z.H. Nazli, 2015. Biomass production and nutritional composition of Moringa oleifera under different cutting frequencies and planting spacings. Int. J. Agric. Biol., 17: 000-000 
2007). Moringa has successfully been grown as field crop for biomass production and high dry matter yields of 4.2 to $8.3 \mathrm{t} \mathrm{ha}^{-1}$ were harvested after every 40 days when planted at different spacing and cutting frequencies (Sanchez et al., 2006). Moringa fresh leaves are rich in crude protein (CP) contents ranging between 19.3-24.3\% (Foidl et al., 1999; Aregheore, 2002). Makkar and Becker (1996, 1997) reported 18747.14 and $1121.00 \mathrm{mg} \mathrm{kg}^{-1} \mathrm{Ca}$ and $\mathrm{P}$ contents, respectively in moringa leaves. In addition, being rich in vitamins, use of moringa can make up the nutritional deficiency in livestock and human beings (Nambiar, 2006).

The biomass production and cultural practices have been discussed in other parts of the world as a fodder crop but a little emphasis was given on this topic in Pakistan. The researchers have reported different yields of moringa fodder under different climatic condition as affected by cutting height, cutting intervals, planting pattern and geometry (Scanchez et al., 2006; Mendieta-Araica et al., 2012) while no study is available on this aspect under climatic conditions of Pakistan. The present study was conducted with the objective to optimize the cutting interval at different plant spacing for maximum biomass with better nutritional quality for sustainable production of livestock during dry season with fodder shortage.

\section{Materials and Methods}

\section{Experimental Design and Plant Material}

A field experiment was conducted at Agronomic Research Area, University of Agriculture Faisalabad, Pakistan (latitude $31.3 \mathrm{~N}^{\circ}$, longitude $71.03 \mathrm{E}^{\circ}$, and altitude $184 \mathrm{~m}$ from sea level) during 2009 and 2010. The experiment was laid out in randomized complete block design with factorial arrangement using three replications. Seeds of moringa collected from trees located at Agronomy Research Area University of Agriculture, Faisalabad were used as experimental material.

\section{Crop Husbandry}

Seed beds of 30 and $60 \mathrm{~cm}$ width were prepared with tractor drawn ridger. The crop was sown on September 6, 2009 and cutting height was maintained to continue for the next growing seasons. Moringa seeds were planted at $2 \mathrm{~cm}$ depth at two plant spacing i.e. $\mathrm{P}_{1}=15 \times 30 \mathrm{~cm}$ and $\mathrm{P}_{2}=15 \times 60$ $\mathrm{cm}$ with hand dibbler. One month after their emergence, moringa plants were given a uniformity cut at $10 \mathrm{~cm}$ height. Later, three cutting intervals i.e., 15, 20 and $30 \mathrm{~d}$ were employed in this study. Soil physico-chemical analysis conducted at Soil Chemistry Lab., Institute of Soil and Environmental Sciences indicated that it was sandy clay and slightly alkaline with $\mathrm{EC}_{\mathrm{e}} 0.39 \mathrm{dS} \mathrm{m} \mathrm{m}^{-1}, \mathrm{pH} 7.4$ with exchangeable $\mathrm{Na}^{+} 0.23$ mmolc $100 \mathrm{~g}^{-1}$, low in organic matter $0.91 \%, \mathrm{~N} 0.091 \%, \mathrm{P} 6.34 \mathrm{mg} \mathrm{kg}^{-1}$, medium in $179 \mathrm{mg} \mathrm{kg}^{-1}$ exchangeable $\mathrm{K}$ and with adequate levels of $\mathrm{B}, \mathrm{Zn}$ and $\mathrm{Fe}$
(0.83, 1.29 and $7.14 \mathrm{mg} \mathrm{kg}^{-1}$, respectively). Nitrogen was applied $90 \mathrm{~kg} \mathrm{ha}^{-1}$ as basal dose using urea as a source. Weeds were controlled manually and crop was irrigated weekly during early crop growth period and thereafter at fortnight interval. Seed emergence was counted according to standard procedures.

\section{Sampling Procedure}

For stand establishment, numbers of emerged seeds were counted daily according to the seedling evaluation handbook of AOSA (1990). Time taken to 50\% emergence $\left(\mathrm{E}_{50}\right)$ and mean emergence time (MET) was calculated according to equation of Ellis and Roberts (1981). Emergence index (EI) was calculated following the formula given by AOSA (1983) and final emergence was calculated as total number of seeds emerged at final count and expressed in percentage. Then seedlings were allowed to grow till $30 \mathrm{~cm}$ height and plants were harvested manually at $10 \mathrm{~cm}$ from ground level after four months of sowing. Later on, plants were allowed to grow until the sprouting started in late February and attained optimum ground cover in April 2010. The first harvest was obtained on 15 April, 2010 from all treatments and second harvest after subsequent interval of 20,30 and $15 \mathrm{~d}$. Number of branches per plant was counted from ten randomly tagged and selected plants at each and every harvest.

For biomass production, at each cutting interval, fresh weight from unit area $\left(\mathrm{m}^{2}\right)$ randomly selected plants was taken immediately after each harvest and averaged. For dry weight, harvested plants were oven-dried at $70 \pm 2{ }^{\circ} \mathrm{C}$ in EYLA Forced air oven (WFO-600 ND, Rikakikai Co. Ltd. Tokyo, Japan) till constant weight was achieved using. Two harvests were taken each year i.e., 2009 and 2010.

\section{Nutrient Composition Determination}

During 2009, leaves from both harvest of each cutting interval were subjected for determination nutrient composition only. For this purpose, leaves of similar age from selected plants were collected randomly and analyzed for $\mathrm{N}, \mathrm{P}, \mathrm{K}, \mathrm{Ca}$, and ascorbic acid concentration. About 50 leaf samples were collected from each moringa plant to analyze nutrient status.

From each replication, 4 to 7 healthy leaves from terminal fully expanded branches without any deficiency symptoms or injury by insects and disease were collected carefully at each cutting during second year of the research. Leaves with petioles were given washings with distilled water 2-3 times and finally rinsed. Afterwards, leaves were dried under shade for $48 \mathrm{~h}$ and oven-dried at $70 \pm 2{ }^{\circ} \mathrm{C}$ till constant weight. Then dried leaf samples were grinded to fine powder in an electric stainless steel grinder to pass through $2 \mathrm{~mm}$ sieve. The grinded leaf powder was stored after labelling in air tight plastic bags at room temperature for further processing. 
Total nitrogen $(\mathrm{N})$ contents were quantified by Chapman and Pratt (1961) method and for total phosphorous $(\mathrm{P})$, potassium $(\mathrm{K})$ and calcium $(\mathrm{Ca})$ contents were determined by following the method described by Rashid (1986). P contents were quantified on UVspectrophotometer at $410 \mathrm{~nm}$ wavelength. Flame photometer (Jenway PEP-7) was used to determine $\mathrm{K}$ contents (Chapman and Pratt, 1961) while $\mathrm{Ca}$ was determined by atomic absorption spectrophotometer (Model: Z-8200; Hitachi, Japan).

\section{Ascorbate Content Estimation}

Ascorbate contents in moringa leaves were quantified by following the procedure devised by Intrigliolo et al. (1999). Ascorbic acid was used as reference standard with stock of $100 \mu \mathrm{g} \mathrm{mL}^{-1}$ and from stock solution, 2, 4, 6, 8 and $10 \mathrm{~mL}$ solution was taken to prepare five standards of $20,40,60$, 80 and $100 \mu \mathrm{g} \mathrm{mL}^{-1}$ to draw the standard curve ( $\mathrm{Y}=0.0202 \mathrm{x}$ $+0.1937)$. The absorbance measured was fitted in standard curve to calculate ascorbic acid contents in moringa leaves.

\section{Statistical Analysis}

The experiment was conducted in randomized completely block design (RCBD) with two factor factorial arrangement. The data were pooled and analyzed using MSTAT-C Program (MSTAT Development Team, 1989). LSD test at $5 \%$ level of probability was used to test the differences among mean values (Steel et al., 1996).

\section{Results}

No significant difference was recorded in emergence speed and final emergence percentage of moringa seeds planted at $15 \times 30 \mathrm{~cm}$ and $15 \times 60 \mathrm{~cm}$ spacings (Table 1). Plant biomass yield of moringa crop was significantly affected by plant spacing, cutting interval and their interaction in both years. During research period (2009 and 2010), highest fresh and dry biomass yield of moringa crop was recorded at narrow planting spacing with $30 \mathrm{~d}$ cutting interval of $30 \mathrm{~d}$ while the interaction of planting spacing and cutting interval was non-significant at $2^{\text {nd }}$ harvest in case of fresh and dry biomass. Moreover, moringa crop planted at wider spacing produced lowest yield at $20 \mathrm{~d}$ cutting interval (Table 2 and 3 ). The number of branches per plant was not affected by the interaction of both factors i.e., spacing and cutting interval in $1^{\text {st }}$ year of research while crop harvested at $30 \mathrm{~d}$ cutting interval produced more number of branches than others (Table 2). A significant reduction in number of branches was observed in moringa plants when the crop was planted at narrow spacing and harvested at 15 and $20 \mathrm{~d}$ cutting interval in $2^{\text {nd }}$ year of research (Table 3 ).

Nutrient composition of moringa leaves significantly $(\mathrm{p}<0.05)$ varied with cutting intervals at both harvests established by narrow and wider plant spacing. Maximum leaf $\mathrm{N}$ content were recorded for both harvests at wider spacing with $30 \mathrm{~d}$ cutting interval followed by narrow spacing with $15 \mathrm{~d}$ cutting interval. Leaf $\mathrm{P}$ was highest in moringa plants established at narrow spacing with $20 \mathrm{~d}$ cutting interval at $1^{\text {st }}$ harvest, while in $2^{\text {nd }}$ harvest, maximum leaf $\mathrm{P}$ was found in wider planted moringa crop at $15 \mathrm{~d}$ cutting interval. Maximum leaf $\mathrm{K}$ was recorded for wider spacing harvested at $30 \mathrm{~d}$ cutting interval at both harvests, which was followed by wider spacing with $15 \mathrm{~d}$ cutting interval at $1^{\text {st }}$ harvest and narrow spacing with $20 \mathrm{~d}$ interval at $2^{\text {nd }}$ harvest. Leaf ascorbate content showed the same trend while this content was statistically similar to narrow spacing with cutting interval of $20 \mathrm{~d}$ at $2^{\text {nd }}$ harvest and response was non-significant at $1^{\text {st }}$ harvest (Table 4).

Crop growth rate (CGR) was highest in moringa plants established at narrow spacing with cutting interval of $15 \mathrm{~d}$ at both harvests which was followed by $30 \mathrm{~d}$ cutting interval at both narrow and wider spacing in $1^{\text {st }}$ harvest. In $2^{\text {nd }}$ harvest, both 20 and $30 \mathrm{~d}$ cutting intervals showed a similar growth rate (Fig. 1).

\section{Discussion}

Leaf harvesting from fodder trees has been practised since ancient times for being used especially in rainy and summer seasons (Stür et al., 1994). In the present study, the potential of moringa crop for producing better yield with good chemical composition was studied under different planting spacings and cutting intervals. Maximum fresh and dry biomass was harvested from moringa plants grown at narrow spacing with cutting interval of $30 \mathrm{~d}$ while a reduction was observed at higher cutting intervals. A positive correlation between planting spacings and yield has been reported in literature for various plant species like Sesbania grandiflora, Leucaena leucocephala and Gliricidia sepium (Ella et al., 1989; Blair et al., 1990; Stür et al., 1994). At narrow spacing, the plants compete for sunlight, nutrients and water uptake, which can result in reduced yield per plant while this reduction can be balanced by total yield per unit area (Ella et al., 1989; Norman, 1992). In present study, moringa crop planted at narrow spacing provided higher biomass in comparison with wider ones. Foidl et al. (2001) reported a positive correlation between increasing planting density and total moringa yield while Sanchez et al. (2006) reported statistically significant variation in moringa yield under different planting densities. Mendiata-Araica et al. (2012) also reported an increased yield at narrow spacing $\left(167,000\right.$ plants $\left.^{-1} \mathrm{a}^{-1}\right)$ in comparison with wider spacing $\left(100,000\right.$ plant $\left.\mathrm{ha}^{-1}\right)$. It was also observed in this study that maximum yield was obtained when moringa crop was harvested at $30 \mathrm{~d}$ cutting interval as explained above. Sanchez et al. (2006) reported higher biomass yield at $75 \mathrm{~d}$ cutting interval but employed 45-75 d cutting intervals due to different climatic conditions of study site located in Nicaragua. Longer cutting intervals produces less fine fraction of yield (woody biomass increases), 
Table 1: Stand establishment of moringa at different planting spacing

\begin{tabular}{lllll}
\hline Planting Spacing & FEP $(\%)$ & MET $(\mathrm{d})$ & $\mathrm{E}_{50}(\mathrm{~d})$ & EI \\
\hline$(15 \times 30 \mathrm{~cm})$ & $27.09 \pm 1.84$ & $11.58 \pm 0.32$ & $11.23 \pm 0.66$ & $11.20 \pm 0.66$ \\
$(15 \times 60 \mathrm{~cm})$ & $25.40 \pm 0.92$ & $11.41 \pm 0.456$ & $10.69 \pm 0.75$ & $10.34 \pm 0.75$ \\
\hline Final emergence percentage $\left(\right.$ FEP), mean emergence time $(\mathrm{MET})$, time taken for 50\% emergence $\left(\mathrm{E}_{50}\right)$ and emergence index (EI)
\end{tabular}

Table 2: Biomass yield of moringa as affected by plant spacing and cutting intervals during 2009

\begin{tabular}{|c|c|c|c|c|c|c|}
\hline \multirow{2}{*}{$\begin{array}{l}\text { Treatments/ } \\
\text { Harvests }\end{array}$} & \multicolumn{2}{|c|}{ Fresh matter yield $\left(\mathrm{t} \mathrm{ha}^{-1}\right)$} & \multicolumn{2}{|c|}{ Dry matter yield $\left(\mathrm{t} \mathrm{ha}^{-1}\right)$} & \multicolumn{2}{|c|}{ No. of Branches per plant } \\
\hline & $\overline{1^{\text {st }}}$ & $2^{\text {nd }}$ & $1^{\mathrm{st}}$ & $2^{\text {nd }}$ & $1^{\mathrm{st}}$ & $2^{\text {nd }}$ \\
\hline \multicolumn{7}{|l|}{ Plant spacing $(\mathrm{P})$} \\
\hline$P_{1}(15 \times 30 \mathrm{~cm})$ & $4.88 \mathrm{a}$ & $5.46 \mathrm{a}$ & $1.10 \mathrm{a}$ & $1.12 \mathrm{a}$ & 9.98 & 10.00 \\
\hline$P_{2}(15 \times 60 \mathrm{~cm})$ & $3.95 \mathrm{~b}$ & $4.45 \mathrm{~b}$ & $0.90 \mathrm{~b}$ & $1.01 \mathrm{~b}$ & 10.02 & 9.83 \\
\hline$L S D$ & 0.20 & 0.22 & 0.03 & 0.06 & n.s. & n.s. \\
\hline \multicolumn{7}{|c|}{ Cutting Intervals (C) } \\
\hline $\mathrm{C}_{1}(15 \mathrm{~d})$ & $5.09 \mathrm{~b}$ & $5.51 \mathrm{~b}$ & $1.38 \mathrm{~b}$ & $1.17 \mathrm{a}$ & $9.33 \mathrm{~b}$ & 9.85 \\
\hline $\mathrm{C}_{2}(20 \mathrm{~d})$ & $2.37 \mathrm{c}$ & $3.50 \mathrm{c}$ & $0.54 \mathrm{c}$ & $0.82 \mathrm{~b}$ & $9.80 \mathrm{~b}$ & 9.90 \\
\hline $\mathrm{C}_{3}(30 \mathrm{~d})$ & $5.79 \mathrm{a}$ & $5.84 \mathrm{a}$ & $1.33 \mathrm{a}$ & $1.21 \mathrm{a}$ & $10.87 \mathrm{a}$ & 10.00 \\
\hline$L S D$ & 0.25 & 0.22 & 0.04 & 0.058 & 0.86 & n.s. \\
\hline \multicolumn{7}{|c|}{ Interaction $(\mathrm{P} \times \mathrm{C})$} \\
\hline $\mathrm{P}_{1} \mathrm{C}_{1}$ & $5.60 \mathrm{~b}$ & 6.07 & $1.23 \mathrm{~b}$ & 0.22 & 9.27 & 10.00 \\
\hline $\mathrm{P}_{1} \mathrm{C}_{2}$ & $2.63 \mathrm{e}$ & 4.11 & $0.59 \mathrm{~d}$ & 0.86 & 9.33 & 10.00 \\
\hline $\mathrm{P}_{1} \mathrm{C}_{3}$ & $6.40 \mathrm{a}$ & 6.20 & $1.48 \mathrm{a}$ & 1.29 & 11.33 & 10.00 \\
\hline $\mathrm{P}_{2} \mathrm{C}_{1}$ & $4.57 \mathrm{~d}$ & 4.96 & $1.04 \mathrm{c}$ & 1.12 & 9.40 & 9.70 \\
\hline $\mathrm{P}_{2} \mathrm{C}_{2}$ & $2.09 \mathrm{f}$ & 2.89 & $0.49 \mathrm{e}$ & 0.78 & 10.27 & 9.80 \\
\hline $\mathrm{P}_{2} \mathrm{C}_{3}$ & $5.19 \mathrm{c}$ & 5.48 & $1.19 \mathrm{~b}$ & 1.13 & 10.40 & 10.00 \\
\hline$L S D$ & 0.35 & n.s. & 0.06 & n.s. & n.s. & n.s. \\
\hline
\end{tabular}

Mean sharing the same letter in a column do not differ significantly at $\mathrm{P}=0.05 ;$ n.s. $=$ Non-significant

Table 3: Biomass yield of moringa as affected by plant spacing and cutting intervals during 2010

\begin{tabular}{|c|c|c|c|c|c|c|}
\hline \multirow{2}{*}{$\begin{array}{l}\text { Treatments/ } \\
\text { Harvests }\end{array}$} & \multicolumn{2}{|c|}{ Fresh matter yield $\left(\mathrm{t} \mathrm{ha}^{-1}\right)$} & \multicolumn{2}{|c|}{ Dry matter yield $\left(\mathrm{t} \mathrm{ha}^{-1}\right)$} & \multicolumn{2}{|c|}{ No. of Branches per plant } \\
\hline & $1^{\mathrm{st}}$ & $2^{\text {nd }}$ & $1^{\mathrm{st}}$ & $2^{\text {nd }}$ & $1^{\mathrm{st}}$ & $2^{\text {nd }}$ \\
\hline \multicolumn{7}{|l|}{$\overline{\text { Plant spacing }(\mathrm{P})}$} \\
\hline $\mathrm{P}_{1}(15 \times 30 \mathrm{~cm})$ & $6.25 \mathrm{a}$ & $4.37 \mathrm{a}$ & $1.60 \mathrm{a}$ & $1.01 \mathrm{a}$ & $9.78 \mathrm{a}$ & $7.33 \mathrm{a}$ \\
\hline $\mathrm{P}_{2}(15 \times 60 \mathrm{~cm})$ & $3.58 \mathrm{~b}$ & $1.82 \mathrm{~b}$ & $0.95 \mathrm{~b}$ & $0.45 \mathrm{~b}$ & $9.22 \mathrm{~b}$ & $6.78 \mathrm{~b}$ \\
\hline$L S D$ & 0.10 & 0.49 & 0.027 & 0.02 & 0.54 & 0.41 \\
\hline \multicolumn{7}{|c|}{ Cutting Intervals (C) } \\
\hline$C_{1}(15 d)$ & $4.02 \mathrm{c}$ & $3.32 \mathrm{~b}$ & $1.28 \mathrm{~b}$ & $0.79 \mathrm{a}$ & $8.00 \mathrm{c}$ & $7.17 \mathrm{~b}$ \\
\hline$C_{2}(20 d)$ & $4.92 \mathrm{~b}$ & $1.96 \mathrm{c}$ & $1.13 \mathrm{c}$ & $0.59 \mathrm{~b}$ & $9.50 \mathrm{~b}$ & $4.50 \mathrm{~b}$ \\
\hline $\mathrm{C}_{3}(30 \mathrm{~d})$ & $5.79 \mathrm{a}$ & $4.01 \mathrm{a}$ & $1.41 \mathrm{a}$ & $0.81 \mathrm{a}$ & $11.00 \mathrm{a}$ & $9.50 \mathrm{a}$ \\
\hline$L S D$ & 0.12 & 0.06 & 0.33 & 0.03 & 0.66 & 0.51 \\
\hline \multicolumn{7}{|c|}{ Interaction $(\mathrm{P} \times \mathrm{C})$} \\
\hline $\mathrm{P}_{1} \mathrm{C}_{1}$ & $5.15 \mathrm{c}$ & $4.88 \mathrm{~b}$ & $1.67 \mathrm{~b}$ & $1.03 \mathrm{~b}$ & $8.67 \mathrm{c}$ & $7.33 \mathrm{~b}$ \\
\hline $\mathrm{P}_{1} \mathrm{C}_{2}$ & $6.02 \mathrm{~b}$ & $2.79 \mathrm{c}$ & $1.34 \mathrm{c}$ & $0.78 \mathrm{c}$ & $9.33 \mathrm{bc}$ & $5.00 \mathrm{c}$ \\
\hline $\mathrm{P}_{1} \mathrm{C}_{3}$ & $7.57 \mathrm{a}$ & $5.44 \mathrm{a}$ & $1.79 \mathrm{a}$ & $1.24 \mathrm{a}$ & $11.33 \mathrm{a}$ & $9.67 \mathrm{a}$ \\
\hline $\mathrm{P}_{2} \mathrm{C}_{1}$ & $2.90 \mathrm{f}$ & $1.77 \mathrm{e}$ & $0.89 \mathrm{e}$ & $0.57 \mathrm{~d}$ & $7.33 \mathrm{~d}$ & $7.00 \mathrm{~b}$ \\
\hline $\mathrm{P}_{2} \mathrm{C}_{2}$ & $3.81 \mathrm{e}$ & $1.12 \mathrm{f}$ & $0.92 \mathrm{e}$ & $0.39 \mathrm{e}$ & $9.67 \mathrm{~b}$ & $4.00 \mathrm{~d}$ \\
\hline $\mathrm{P}_{2} \mathrm{C}_{3}$ & $4.03 \mathrm{~d}$ & $2.58 \mathrm{~d}$ & $1.03 \mathrm{~d}$ & $0.39 \mathrm{e}$ & $10.67 \mathrm{a}$ & $9.33 \mathrm{a}$ \\
\hline$L S D$ & 0.18 & 0.08 & 0.05 & 0.52 & 0.94 & 0.72 \\
\hline
\end{tabular}

Mean sharing the same letter in a column do not differ significantly at $\mathrm{P}=0.05$; n.s. = Non-significant

so $75 \mathrm{~d}$ cutting interval is not suitable in climatic condition of the present study site. So, the intervals were set at 15, 20 and 30 days, which suited better for such climatic conditions as moringa is indigenous to Punjab and flourish better under these condition. Secondly, if cutting interval increases, woody branches can shade lower branches, which can affect the photosynthesis process that might result in reduction in total yield (Newton et al., 2006). Latt et al. (2000) reported that frequent harvesting of plants reduces the nutrient assimilation which affects the growth rate of plants by affecting the leaf development as observed for $15 \mathrm{~d}$ cutting interval of present study (Table 2 and 3). Assefa (1998) suggested keeping suitable cutting interval giving time to plants to regenerate and absorb the cutting shock.

Planting density affects the leaf mineral composition (Newton et al., 2006). In this study, a decrease in leaf chemical composition was recorded with increase in planting density. Moringa plants planted at narrow spacing provided higher leaf nutrients in comparison with the wider ones (Table 4). Sanchez et al. (2006) reported that mineral composition of moringa plants decreases with increasing planting density. However, El-Morsy (2009) reported a 
Table 4: Chemical composition of total digestible nutrients in moringa as affected by plant spacing and cutting intervals

\begin{tabular}{|c|c|c|c|c|c|c|c|c|c|c|}
\hline \multirow{2}{*}{$\begin{array}{l}\text { Treatments/ } \\
\text { Harvests }\end{array}$} & \multicolumn{2}{|c|}{ Nitrogen $(\%)$} & \multicolumn{2}{|c|}{ Phosphorus (\%) } & \multicolumn{2}{|c|}{ Potassium (\%) } & \multicolumn{2}{|c|}{ Calcium (\%) } & \multicolumn{2}{|c|}{ Ascorbate $\left(\mu \mathrm{g} \mathrm{g}^{-1}\right)$} \\
\hline & $\overline{1^{\mathrm{st}}}$ & $2^{\text {nd }}$ & $1^{\mathrm{st}}$ & $2^{\text {nd }}$ & $1^{\mathrm{st}}$ & $2^{\text {nd }}$ & $1^{\mathrm{st}}$ & $2^{\text {nd }}$ & $1^{\mathrm{st}}$ & $2^{\text {nd }}$ \\
\hline \multicolumn{11}{|l|}{ Plant spacing (P) } \\
\hline $\mathrm{P}_{1}(15 \times 30 \mathrm{~cm})$ & $5.87 \mathrm{a}$ & $5.99 \mathrm{a}$ & $3.28 \mathrm{a}$ & 1.80 & $7.59 \mathrm{a}$ & 7.30 & 2.22 & 2.04 & $87.88 \mathrm{a}$ & 76.88 \\
\hline$P_{2}(15 \times 60 \mathrm{~cm})$ & $5.66 \mathrm{~b}$ & $5.84 \mathrm{~b}$ & $1.57 \mathrm{~b}$ & 1.16 & $6.66 \mathrm{~b}$ & 7.30 & 2.47 & 2.13 & $67.05 \mathrm{~b}$ & 78.39 \\
\hline LSD & 0.07 & 0.11 & 0.06 & n.s. & 0.08 & n.s. & n.s. & n.s. & 5.078 & n.s. \\
\hline \multicolumn{11}{|c|}{ Cutting Intervals (C) } \\
\hline $\mathrm{C}_{1}(15 \mathrm{~d})$ & $5.66 \mathrm{~b}$ & $5.78 \mathrm{~b}$ & $2.59 \mathrm{a}$ & $1.80 \mathrm{a}$ & $5.76 \mathrm{c}$ & $6.78 \mathrm{~b}$ & $2.36 \mathrm{a}$ & 2.13 & $77.25 \mathrm{ab}$ & $63.80 \mathrm{c}$ \\
\hline$C_{2}(20 d)$ & $5.67 \mathrm{~b}$ & $5.88 \mathrm{~b}$ & $2.36 \mathrm{~b}$ & $1.16 \mathrm{~b}$ & $7.07 \mathrm{~b}$ & $6.88 \mathrm{~b}$ & $2.27 \mathrm{a}$ & 2.00 & $81.48 \mathrm{a}$ & $89.07 \mathrm{a}$ \\
\hline$C_{3}(30 d)$ & $5.97 \mathrm{a}$ & $6.10 \mathrm{a}$ & $2.31 \mathrm{~b}$ & $0.09 \mathrm{c}$ & $8.56 \mathrm{a}$ & $8.27 \mathrm{a}$ & $2.40 \mathrm{a}$ & 2.13 & $73.66 \mathrm{~b}$ & $80.04 \mathrm{~b}$ \\
\hline LSD & 0.09 & 0.10 & 0.07 & 0.33 & 0.09 & 0.20 & 0.135 & n.s. & 6.22 & 2.38 \\
\hline \multicolumn{11}{|c|}{ Interaction $(\mathrm{P} \times \mathrm{C})$} \\
\hline $\mathrm{P}_{1} \mathrm{C}_{1}$ & $5.82 \mathrm{c}$ & $5.97 \mathrm{~b}$ & $3.35 \mathrm{a}$ & $1.37 \mathrm{bcd}$ & $6.53 \mathrm{~d}$ & $6.82 \mathrm{~d}$ & $2.26 \mathrm{~b}$ & $1.86 \mathrm{c}$ & 85.69 & $71.70 \mathrm{~b}$ \\
\hline $\mathrm{P}_{1} \mathrm{C}_{2}$ & $5.97 \mathrm{~b}$ & $5.97 \mathrm{~b}$ & $3.08 \mathrm{~b}$ & $2.30 \mathrm{a}$ & $8.27 \mathrm{~b}$ & $7.31 \mathrm{c}$ & $2.00 \mathrm{c}$ & $2.00 \mathrm{bc}$ & 92.87 & $88.59 \mathrm{a}$ \\
\hline $\mathrm{P}_{1} \mathrm{C}_{3}$ & $5.83 \mathrm{c}$ & $6.04 \mathrm{ab}$ & $3.40 \mathrm{a}$ & $1.73 \mathrm{~b}$ & $7.98 \mathrm{c}$ & $7.79 \mathrm{~b}$ & $2.40 \mathrm{ab}$ & $2.26 \mathrm{ab}$ & 85.07 & $70.35 \mathrm{~b}$ \\
\hline $\mathrm{P}_{2} \mathrm{C}_{1}$ & $5.50 \mathrm{~d}$ & $5.59 \mathrm{~d}$ & $1.83 \mathrm{c}$ & $1.43 \mathrm{bc}$ & $4.99 \mathrm{f}$ & $6.72 \mathrm{de}$ & $2.47 \mathrm{a}$ & $2.40 \mathrm{a}$ & 68.80 & $55.91 \mathrm{c}$ \\
\hline $\mathrm{P}_{2} \mathrm{C}_{2}$ & $5.38 \mathrm{~d}$ & $5.79 \mathrm{c}$ & $1.63 \mathrm{~d}$ & $0.96 \mathrm{~d}$ & $5.86 \mathrm{e}$ & $6.44 \mathrm{e}$ & $2.53 \mathrm{a}$ & $2.00 \mathrm{bc}$ & 70.09 & $89.54 \mathrm{a}$ \\
\hline $\mathrm{P}_{2} \mathrm{C}_{3}$ & $6.11 \mathrm{a}$ & $6.16 \mathrm{a}$ & $1.23 \mathrm{e}$ & $1.08 \mathrm{~cd}$ & $9.14 \mathrm{a}$ & $8.75 \mathrm{a}$ & $2.40 \mathrm{ab}$ & $2.00 \mathrm{bc}$ & 62.26 & $89.73 \mathrm{a}$ \\
\hline LSD & 0.13 & 0.15 & 0.09 & 0.46 & 0.13 & 0.29 & 0.19 & 0.28 & n.s. & 3.37 \\
\hline
\end{tabular}

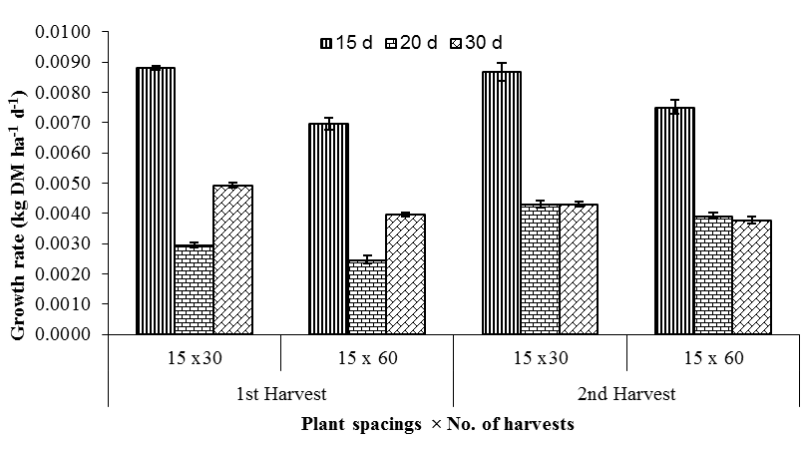

Fig. 1: Growth rate $\left(\mathrm{kg} \mathrm{DM} \mathrm{ha} \mathrm{d}^{-1}\right)$ of moringa as affected by plant spacing and different cutting intervals at two harvests

reduction in nitrogen content in his study with increase in plant population per unit area. This might be attributed to the competition of plants for nutrient uptake. Plants with longer harvest at wider spacing showed maximum $\mathrm{N}$ contents. The difference between findings of these reports and our experiment might be due to difference in experimental and climatic conditions. Likewise, with decreasing cutting intervals, leaf chemical composition is affected as was observed in the present study. Botha and Rethman (1994) who reported a reduction in crude protein and $\mathrm{P}$ contents with reducing cutting intervals. Similarly, Newton et al. (2006) also reported higher mineral contents in moringa leaves at $35 \mathrm{~d}$ cutting interval. It is however difficult to conclude that which factors affect leaf chemical composition of moringa under different cutting intervals.

\section{Conclusion}

Biomass production and nutritional composition of moringa varied with cutting intervals when established at narrow and wider plant spacing. For high biomass yield and better chemical composition, moringa crop should be established at narrow spacing $(15 \times 30 \mathrm{~cm})$ with optimal cutting interval of $30 \mathrm{~d}$. All in all in dry spells, when there is fodder shortage, moringa can be a good source of good quality fodder for livestock.

\section{References}

Abdulkarim, S.M., K. Long, O.M. Lai, S.K.S. Muhammad and H.M Ghazali, 2007. Frying quality and stability of high-oleic Moringa oleifera seed oil in comparison with other vegetable oils. Food Chem., 105: 1382-1389

Association of Official Seed Analysts (AOSA), 1990. Rules for testing seeds. J. Seed Technol., 12: 1-112

Association of Official Seed Analysts (AOSA), 1983. Seed Vigour Testing Handbook. Contribution No. 32 to the handbook on seed testing. Association of Official Seed Analysts. Springfield, Illinois, USA

Aregheore, E.M., 2002. Intake and digestibility of Moringa oleifera-batiki grass mixtures by growing goats. Small Rumin. Res., 46: 23-28

Assefa, G., 1998. Biomass yield, botanical fractions and quality of tagasaste, (Chamaecytisus palmensis) as affected by harvesting interval in the highlands of Ethiopia. Agrofor. Syst., 42: 13-23

Benavides, J.E., 1994. La investigacion de arboles forrajeros. In: Arboles y Arbustos Forrajeros en America Central. Vol. 1, pp: 3-28. Benvides, J.E. (ed.). Turrialba, Costa Rica

Blair, G., D. Catchpoole and P. Horne, 1990. Forage tree legumes: Their management and contribution to the nitrogen economy of wet and humid tropical environments. Adv. Agron., 44: 27-54

Botha, L.T. and N.F.G. Rethman, 1994. Yield and chemical composition of Pennisetum glaucum (L.) at different cutting frequencies. Appl. Plant Sci., 8: $37-42$

Chapman, H.D. and P.F. Pratt, 1961. Methods of Analysis for Soils, Plants and water. University of California, Berkeley, California, USA

El-Morsy, M.H.M., 2009. Influence of cutting height and plant spacing on Sesbania (Sesbania aegyptiaca [Poir]) productivity under hyper-arid conditions in El-kharga Oasis, El-Wadi El-Gaded, Egypt. Int. J. Plant Prod., 3: 77-84

Ella, A., C. Jacobsen C, W. Stür and G. Blair, 1989. Effect of plant density and cutting frequency on the productivity of four tree legumes. Trop. Grassland. 23: 28-34

Ellis, R.A. and E.H. Roberts, 1981. The quantification of ageing and survival in orthodox seeds. Seed Sci. Technol., 9: 373-409

Foidl, N. and R. Paull, 1999. Moringa oleifera. In: The Encyclopedia of Fruit and Nuts, pp: 509-512. CABI, Oxfordshire, UK 
Foidl, N., H.P.S. Makkar and K. Becker, 2001. The potential of Moringa oleifera for agricultural and industrial uses. Proceedings of International Workshop "What Development Potential for Moringa Products?" Dar-es-Salaam, Tanzania

Government of Pakistan, 2014. Pakistan Statistical Year Book, Federal Bureau of statistics, statistics Division Government of Pakistan, Islamabad, Pakistan

HDRA, 2002. Moringa oleifera-A Multipurpose Tree, Produced by the Tropical Advisory Service, HDRA - The Organic Organization. Online available at http://www.hdra.org.uk

Intrigliolo, F., G. Roccuzzo, G. Lacertosa, P. Rapisarda and S. Canali, 1999. Effect of fertilizer on growth and yield of citrus. J. Plant Nutr., 11: 3-7

Latt, C.R., P.K.R. Nair and B.T. Kang, 2000. Interactions among cutting frequency, reserve carbohydrates, and post-cutting biomass production in Gliricidia sepium and Leucaena leucocephala. Agrofor. Syst., 50: 27-46

Makkar, H.P.S. and K. Becker, 1996. Nutritional value and antinutritional components of whole and ethanol extracted Moringa oleifera leaves. Anim. Feed Sci. Technol., 63: 211-228

Makkar, H.P.S. and K. Becker, 1997. Nutrients and antiquality factors in different morphological parts of the Moringa oleifera tree. J. Agric Sci. (Camb.), 128: 311-332

Mendieta-Araica, B., E. Sporndly, N.R.Sanchez, F. S.Miranda and M. Halling, 2012. Biomass production and chemical composition of Moringa oleifera under different planting densities and levels of nitrogen fertilization. Agroforest. Syst., 87- 81-92

MSTAT Development Team, 1989. Mstat user's guide: A microcomputer program for the design management and analysis research experiments. Michigan State Univ. East Lansing, USA

Nambiar, V.S., 2006. Nutritional potential of drumstick leaves: an Indian perspective. Proceedings of moringa and other highly nutritious plant resources: Strategies, standards and markets for a better impact on nutrition in Africa. Accra, Ghana
NARC, 2007. National coordinated fodder research program Islamabad Online available at http://www.parc.gov.pk/fodder.html

Newton, A.K., G.M. Timpo, W.O. Ellis, R.N. Bennett and N. Foidl, 2006 Effect of spacing and harvest frequency on the growth and leaf yield of moringa (Moringa oleifera Lam), a leafy vegetable crop. Ghana J. Hort., 6: 33-40

Norman, J.C., 1992. Tropical Vegetable Crops, pp: 110-252. Arthur H Stockwell Limited, London, UK

Nouman, W., S.M.A. Basra, M.T. Siddiqui, A. Yasmeen, T. Gull, M.A.C. Alcayde, 2014. Potential of Moringa oleifera $\mathrm{L}$. as livestock fodder crop: a review. Turk. J. Agric. For., 38: 1-14

Palada, M.C., 1996. Moringa (Moringa oleifera Lam.): A versatile tree crop with horticultural potential in the subtropical United States. HortSci., 31: 794-797

Pezo, D., 1991. The Nutritional Quality of Forages, p: 15. Producciony utilizacio'n fodder on the throne peak. Compendium. Teaching Materials Series Anza

Rashid, A., 1986. Mapping zinc fertility of soils using indicator plants and soils-analyses. Doctoral Thesis. University of Hawaii, HI, USA

Raziq, A., M. Younas and Z. Rehman, 2010. Prospects of livestock production in Balochistan. Pak. Vet. J., 30: 181-186

Sanchez, N.R., L. Stig and L. Inger, 2006. Biomass Production and Chemical Composition of Moringa oleifera under Different Management Regimes in Nicaragua. Agrofor. Syst., 66: 231-242

Steel, R.C.D., J.H. Torrie and D.A. Deekey, 1996. Principles and Procedures of Statistics a Biometric Approach, $3^{\text {rd }}$ edition, pp: 400-428. McGraw Hill Book Co. Inc. New York, USA

Stür, W.W., H.M. Shelton and R.C. Gutteridge, 1994. Defoliation management of forage tree legumes. In: Forage Tree Legumes in Tropical Agriculture. Gutteridge R.C. and H.M. Shelton (eds). CAB Int. Wallingford, UK

(Received 18 June 2014; Accepted 27 February 2015) 